\title{
Scaling behavior of thin films on chemically heterogeneous walls
}

\author{
Alexandr Malijevský \\ Department of Physical Chemistry, University of Chemical Technology Prague, Praha 6, 166 28, Czech Republic \\ and Institute of Chemical Process Fundamentals of the Czech Academy of Sciences, v. v. i., 16502 Prague 6, Czech Republic \\ Andrew O. Parry \\ Department of Mathematics, Imperial College London, London SW7 2B7, United Kingdom \\ Martin Pospíšil \\ Department of Physical Chemistry, University of Chemical Technology Prague, Praha 6, 166 28, Czech Republic
}

(Received 10 July 2017; published 18 September 2017)

\begin{abstract}
We study the adsorption of a fluid in the grand canonical ensemble occurring at a planar heterogeneous wall which is decorated with a chemical stripe of width $L$. We suppose that the material of the stripe strongly preferentially adsorbs the liquid in contrast to the outer material which is only partially wet. This competition leads to the nucleation of a droplet of liquid on the stripe, the height $h_{m}$ and shape of which (at bulk two-phase coexistence) has been predicted previously using mesoscopic interfacial Hamiltonian theory. We test these predictions using a microscopic Fundamental Measure Density Functional Theory which incorporates shortranged fluid-fluid and fully long-ranged wall-fluid interactions. Our model functional accurately describes packing effects not captured by the interfacial Hamiltonian but still we show that there is excellent agreement with the predictions $h_{m} \approx L^{1 / 2}$ and for the scaled circular shape of the drop even for $L$ as small as 50 molecular diameters. For smaller stripes the droplet height is considerably lower than that predicted by the mesoscopic interfacial theory. Phase transitions for droplet configurations occurring on substrates with multiple stripes are also discussed.
\end{abstract}

DOI: 10.1103/PhysRevE.96.032801

\section{INTRODUCTION}

Complete wetting is a well known example of a surface phase transition occurring at a wall-gas interface in systems for which the contact angle of a liquid drop is zero. For open systems the transition corresponds to the growth in the thickness $\ell_{\text {eq }}$ of an intruding liquid layer which becomes macroscopic as the chemical potential $\mu$ is increased towards its saturation value: $\delta \mu=\mu_{\text {sat }}-\mu \rightarrow 0$. Studies of complete wetting date back to the Russian school of Derjaguin [1] who formulated the problem using the concept of a disjoining pressure and were the first to recognize the importance of microscopic forces in determining the growth of the film thickness. This phase transition was subsequently rediscovered and placed within the greater context of wetting transitions and fluid interfacial phenomena following the seminal works of Cahn [2] and Ebner and Saam [3] in the 1970s. Consequently over the last three decades there have been numerous theoretical (and experimental) studies of complete wetting at planar, and chemically homogeneous, substrates using the modern statistical mechanical theory of inhomogeneous fluids; for reviews see, for example, [4-8]. Of particular importance in the theoretical development have been treatments based on microscopic density functional theory (DFT) [9] and interfacial Hamiltonian approaches which allow for the influence of interfacial fluctuations [10]. These studies have highlighted that in addition to the growth of $\ell_{\text {eq }}$, complete wetting is also characterised by the divergence of a parallel correlation length $\xi_{\|}$due to (thermal) fluctuations of the liquidgas interface as it unbinds from the wall. Renormalization group studies of interfacial models show for systems with long-ranged forces the upper critical dimension for complete wetting $d^{*}<3$, meaning that mean-field treatments, including the predictions of the original Derjaguin theory, are correct in three dimensions. More specifically, for systems with longrange attractive molecular forces decaying asymptotically as $r^{-p-4}$, the wetting film $\ell_{\mathrm{eq}}$ and parallel correlation length $\xi_{\|}$ diverge according to the power-laws [10]

$$
\ell_{\mathrm{eq}} \sim \delta \mu^{-\beta^{\mathrm{co}}}, \quad \xi_{\|} \sim \delta \mu^{-\nu_{\|}^{\mathrm{co}}}
$$

with $\beta^{\text {co }}=1 /(p+1)$ and $v_{\|}^{\text {co }}=(p+2) / 2(p+1)$. Thus for systems with non-retarded van der Waals forces $(p=2)$, the values of the critical exponents are $\beta^{\text {co }}=1 / 3$ and $\nu_{\|}^{\text {co }}=2 / 3$, respectively.

More recently, similar theoretical tools have been used to study the adsorption and wetting on both heterogeneous (chemically patterned) and structured solid substrates. This research has revealed a number of novel interfacial phenomena, not present for planar homogeneous substrates, including new phase transitions and enhanced fluctuation effects. These and related studies are also of practical relevance to nanotechnologies involving the fabrication of functional surfaces which control the adsorption of microscopically small amounts of liquid [11]. For geometrically sculpted substrates, theoretical studies of wedges [12-14], grooves [15-17] and cones [18] have shown that the wall geometry can dramatically alter the adsorption properties and accompanying interfacial fluctuation effects. New transitions also arise for planar but chemically heterogeneous substrates in which the wall is a composite formed by materials with different wetting properties. These include studies of unbending transitions [19-21] involving the local condensation of liquid within a patterned region, and also complete pre-wetting (also called step-wetting) in which a nucleated liquid phase spreads out laterally across the substrate $[22,23]$. It is even possible to combine chemical patterning and substrate geometry to produces surfaces that are partially wet 
(i.e., have a finite contact angle) even though the materials involved only show complete wetting [24]. In the canonical ensemble, i.e., considering fixed amount of liquid, chemically patterned surfaces also lead to morphological phase transition and the break down of Young's equation [25,26].

In the present work we study fluid adsorption in an open system at a very simple type of chemically heterogeneous substrate; when a single stripe, of finite width $L$ but macroscopic length and depth, of a material that prefers complete wetting is embedded into a surface that is otherwise completely dry (or more generally, partial wet). This contrast leads to the nucleation of a droplet of liquid at the stripe the height $h_{m}$ of which remains finite even at bulk two-phase coexistence $\left(\mu=\mu_{\text {sat }}\right)$. For this system effective Hamiltonian theories make a number of predictions not only for the dependence of $h_{m}$ on $L$ but also for the precise cross-sectional shape of the drop [27]. For systems with short-ranged forces the droplet shape is further predicted to display a conformal invariance which allows its determination on a wide variety of patterned surfaces not just a stripe. For systems with long-ranged forces, pertinent to most solid-fluid interfaces, conformal invariance does not apply in three-dimensions but the droplet height and shape are still predicted to show strong scaling behavior. In the present study we test these specific scaling predictions using an accurate density functional model based on Rosenfeld's fundamental measure theory [28]. In particular, we wish to determine what size of stripe width $L$ is required before the droplet shape agrees with the effective Hamiltonian theory. We note that gravity is unimportant when the stripe width $L$ is considerably smaller than the capillary length (about a $\mathrm{mm}$ ) and is therefore utterly negligible at the microscopic scales studied here.

The remainder of our paper is organised as follows. In Sec. II we derive the net potential for our single-stripe wall assuming that the wall atoms interact with the fluid via a LennardJones 12-6 potential. In Sec. III we present the microscopic DFT model and also recall the interface Hamiltonian theory for the scaling of the drop size and shape. In Sec. IV we present our
DFT results making comparison with the analytic results of the interface Hamiltonian theory. We conclude with a discussion of our results and also highlight possible extension of this work to new phase transitions occurring on multiple stripes.

\section{THE EXTERNAL POTENTIAL OF THE COMPOSITE WALL}

We suppose the composite wall is made from two species, $i=1,2$, each interacting with the fluid particles via the Lennard-Jones 12-6 potential

$$
\phi_{i}(r)=4 \varepsilon_{i}\left[\left(\frac{\sigma}{r}\right)^{12}-\left(\frac{\sigma}{r}\right)^{6}\right],
$$

where the molecular diameter $\sigma$ and also the uniform density distribution $\rho_{w}$ are considered to be identical for both species. The wall occupies the semi-infinite volume $z<0$ and is considered macroscopic and translationally invariant along the $y$ direction. The stripe (species $i=2$ ) occupies the region $0<x<L$ but the whole depth $z<0$ and length $-\infty<y<$ $\infty$ of the wall. The total external potential $V(x, z)$ is independent of $y$ and is obtained by integrating the wall-fluid pair potential over the wall volume from each region. Thus we write

$$
V(x, z)=V_{\infty}(x, z ; 1)+V_{L}(x, z ; 2)+V_{\infty}(L-x, z ; 1) .
$$

The contribution due to outer parts of the wall, formed from species $i=1$, is described by the triple integral

$$
V_{\infty}(x, z ; 1)=\rho_{w} \int_{x}^{\infty} \mathrm{d} x^{\prime} \int_{-\infty}^{\infty} \mathrm{d} y^{\prime} \int_{z}^{\infty} \phi_{1}\left(\sqrt{x^{\prime 2}+y^{\prime 2}+z^{\prime 2}}\right)
$$

This reduces to

$$
V_{\infty}(x, z ; 1)=\pi \varepsilon_{1} \rho_{w} \sigma^{3}\left[\frac{\sigma^{9}}{z^{9}} F_{9}\left(\frac{x}{z}\right)-\frac{\sigma^{3}}{z^{3}} F_{3}\left(\frac{x}{z}\right)\right],
$$

where

$$
F_{9}(\xi)=\frac{2}{45}\left(1+\frac{1}{\xi^{9}}\right)-\frac{1}{2880} \frac{128 \xi^{16}+448 \xi^{14}+560 \xi^{12}+280 \xi^{10}+35 \xi^{8}+280 \xi^{6}+560 \xi^{4}+\xi^{2}+128}{\xi^{9}\left(1+\xi^{2}\right)^{7 / 2}}
$$

and

$$
F_{3}(\xi)=\frac{1}{3}\left[1+\frac{1}{\xi^{3}}-\frac{2 \xi^{4}+\xi^{2}+2}{2 \xi^{3} \sqrt{1+\xi^{2}}}\right] .
$$

The contribution to the potential due to the stripe, formed by species $i=2$, is given by

$$
\begin{aligned}
V_{L}(x, z ; 2)= & \rho_{w} \int_{x-L}^{L} \mathrm{~d} x^{\prime} \int_{-\infty}^{\infty} \mathrm{d} y^{\prime} \int_{z}^{\infty} \mathrm{d} z^{\prime} \phi_{2} \\
& \times\left(\sqrt{x^{\prime 2}+y^{\prime 2}+z^{\prime 2}}\right) .
\end{aligned}
$$

which has now, owing to the finite size character of the stripe, the scaling form

$$
V_{L}(x, z ; 2)=\pi \varepsilon_{2} \rho_{w} \sigma^{3}\left[\frac{\sigma^{9}}{z^{9}} G_{9}\left(\frac{x}{z}, \frac{L}{z}\right)-\frac{\sigma^{3}}{z^{3}} G_{3}\left(\frac{x}{z}, \frac{L}{z}\right)\right]
$$

with

$$
G_{9}(\xi, \eta)=F_{9}(\xi-\eta)-F_{9}(\xi)
$$

and

$$
G_{3}(\xi, \eta)=F_{3}(\xi-\eta)-F_{3}(\xi) .
$$

Note that in the limit of $L \rightarrow \infty$, the attractive part of the wall potential decays as

$$
V(x, z) \sim-\frac{2}{3} \pi \varepsilon_{2} \rho_{w} \sigma^{3}\left(\frac{\sigma}{z}\right)^{3}
$$

recovering the behavior expected for a planar three-dimensional homogeneous wall. On the other hand for finite $L$ and $z / L \rightarrow \infty$ the wall the potential crosses over to

$$
V(x, z) \sim-\frac{3}{8} \pi \varepsilon_{2} \rho_{w} \sigma^{3}\left(\frac{\sigma}{z}\right)^{3} \frac{L}{z}
$$


which shows the correct power-law appropriate for a pseudo-two-dimensional substrate.

\section{THEORY}

\section{A. Density functional theory}

Within classical density functional theory [29], the equilibrium density profile is obtained from minimising the grand potential functional

$$
\Omega[\rho]=\mathcal{F}[\rho]+\int \mathrm{d} \mathbf{r} \rho(\mathbf{r})[V(\mathbf{r})-\mu],
$$

where $\mu$ is the chemical potential, and $V(\mathbf{r})$ is the external potential due to the wall. The intrinsic free energy functional $\mathcal{F}[\rho]$ is conveniently separated into an exact ideal gas contribution and an excess part:

$$
\mathcal{F}[\rho]=\beta^{-1} \int \mathrm{d} \mathbf{r} \rho(\mathbf{r})\left[\ln \left(\rho(\mathbf{r}) \Lambda^{3}\right)-1\right]+\mathcal{F}_{\text {ex }}[\rho],
$$

where $\Lambda$ is the thermal de Broglie wavelength and $\beta=1 / k_{B} T$ is the inverse temperature. Following the spirit of van der Waals or equivalently simple perturbation theory, the excess part is modelled as a sum of hard-sphere and attractive contributions where the latter is treated in a simple mean-field fashion:

$$
\mathcal{F}_{\text {ex }}[\rho]=\mathcal{F}_{\mathrm{hs}}[\rho]+\frac{1}{2} \iint \operatorname{d} \mathbf{r} \mathrm{d} \mathbf{r}^{\prime} \rho(\mathbf{r}) \rho\left(\mathbf{r}^{\prime}\right) u_{\mathrm{a}}\left(\left|\mathbf{r}-\mathbf{r}^{\prime}\right|\right),
$$

where $u_{\mathrm{a}}(r)$ is the attractive part of the fluid-fluid interaction potential. In our model the fluid atoms are assumed to interact with one another via the truncated (i.e., short-ranged) and non-shifted Lennard-Jones-like potential

$$
u_{\mathrm{a}}(r)= \begin{cases}0 ; & r<\sigma, \\ -4 \varepsilon\left(\frac{\sigma}{r}\right)^{6} ; & \sigma<r<r_{c}, \\ 0 ; & r>r_{c} .\end{cases}
$$

which is cut-off at $r_{c}=2.5 \sigma$, where $\sigma$ is the hard-sphere diameter.

The hard-sphere part of the excess free energy is approximated using the Fundamental Measure Theory functional [28],

$$
\mathcal{F}_{\mathrm{hs}}[\rho]=\frac{1}{\beta} \int \mathrm{d} \mathbf{r} \Phi\left(\left\{n_{\alpha}\right\}\right),
$$

which accurately takes into account short-range correlations between fluid particles and is known to satisfy exact statistical mechanical sum rules [30]. Here, the free energy density $\Phi$ depends on a set of six weighted densities $\left\{n_{\alpha}(\mathbf{r})\right\}$ which may be expressed as double integrals over the $x$ and $z$ dimensions [31]. These are evaluated numerically using Gaussian quadrature.

Minimization of Eq. (14) leads to the Euler-Lagrange equation

$$
V(\mathbf{r})+\frac{\delta \mathcal{F}_{\mathrm{hs}}[\rho]}{\delta \rho(\mathbf{r})}+\int \mathrm{d} \mathbf{r}^{\prime} \rho\left(\mathbf{r}^{\prime}\right) u_{\mathrm{a}}\left(\left|\mathbf{r}-\mathbf{r}^{\prime}\right|\right)=\mu,
$$

which can be solved iteratively on an appropriately discretized two dimensional grid with suitable boundary conditions (see later).

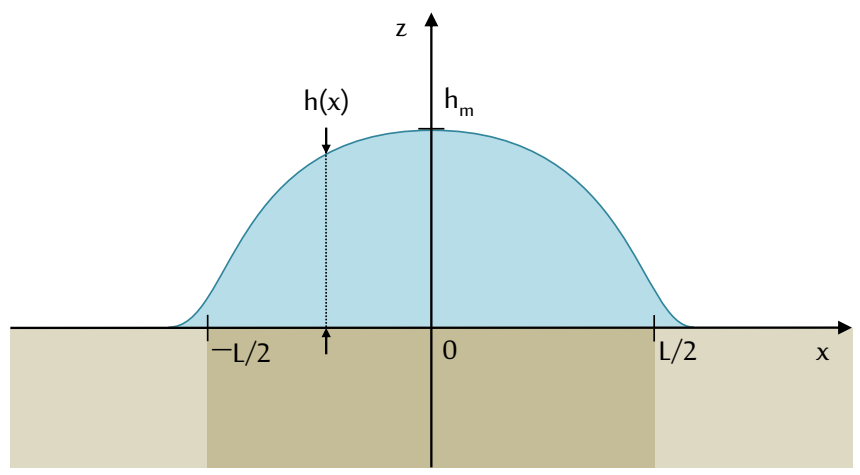

FIG. 1. Schematic illustration of the shape of a drop, of height $h_{m}$, above a composite planar wall containing stripe of material, of finite width $L$ but infinite depth and length, which is completely wet. The outer parts of the substrate only show microscopic adsorption corresponding to partial wetting (or indeed drying).

\section{B. Interfacial Hamiltonian theory}

An alternative and complementary approach to microscopic DFT, applicable to interfacial phenomena occurring at the mesoscopic scale is based on the analysis of simple effective Hamiltonian models. These have been successfully applied to the theory of wetting at planar (homogeneous) walls [5,9] and also continuous wedge filling [13] providing a complete classification of universality classes and scaling regimes arising due to the interplay between fluctuation effects and those directly coming from the intermolecular forces. In application to the present heterogeneous wall, the very simplest effective Hamiltonian that can be used is given by the model [27]

$$
H[h]=\int \mathrm{d} x\left\{\frac{\gamma}{2}\left(\frac{d h}{d x}\right)^{2}+W(h)\right\},
$$

describing the functional dependence of the free-energy (per unit wall length) on the height $h(x)$ of the liquid-vapor interface $h(x)$ above the stripe (see Fig. 1). Here, $\gamma$ is the liquid-vapor surface tension and $W(h)$ is the binding potential describing the complete wetting properties which we specify below. At mean field level the equilibrium configuration of the interface is be determined by simply minimising the functional (20), yielding the Euler-Lagrange equation

$$
\gamma \frac{d^{2} h}{d x^{2}}=W^{\prime}(h) .
$$

This must be solved subject to boundary condition $h(|L| / 2)=$ $h_{e}$, where the edge value $h_{e}$ is considered fixed and microscopic.

Within the model (20) the width of the stripe $L$ is assumed to be large enough that the binding potential can be considered to be independent of $x$ and approximated by the same function appropriate to wetting at planar homogeneous wall. In the case of the Lennard-Jones wall-fluid interaction the binding potential, at bulk saturation, takes the form

$$
W(h)=\frac{A}{h^{2}},
$$


where $A=\pi \varepsilon_{2} \rho_{w} \sigma^{6}\left(\rho_{l}-\rho_{v}\right) / 3$ is the Hamaker constant associated with the wettable part of the wall and $\rho_{l}$ and $\rho_{v}$ are the liquid and vapor saturated densities, respectively. Substituting Eq. (22) into Eq. (21) and integrating once we obtain (for $x<0$ )

$$
\frac{d h(x)}{d x}=\sqrt{\frac{2 A}{\gamma}} \frac{\sqrt{h_{m}^{2}-h^{2}(x)}}{h_{m} h(x)}
$$

and with a change of sign for $x>0$. Here, $h_{m}$ is the maximum of $h(x)$ occurring at $x=0$. Further integration leads to

$$
\sqrt{h_{m}^{2}-h^{2}(x)}=\sqrt{\frac{2 A}{\gamma}} \frac{x}{h_{m}},
$$

and hence from the boundary condition

$$
\sqrt{h_{m}^{2}-h_{e}^{2}}=\sqrt{\frac{2 A}{\gamma}} \frac{L}{2 h_{m}} .
$$

Assuming that $h_{m} \gg h_{e}$ we obtain the simple power-law dependence of the droplet height on the stripe width

$$
h_{m}^{2} \approx L \sqrt{\frac{A}{2 \gamma}} .
$$

which is independent of $h_{e}$ and hence on the properties of the outer wall (provided these remain partial wet). Substituting Eq. (26) into Eq. (24) then implies that the droplet height has a simple universal elliptical shape

$$
\tilde{h}(x)=\sqrt{1-4 \tilde{x}^{2}},
$$

when expressed in rescaled variables $\tilde{x}=x / L$ and $\tilde{h}(x)=$ $h(x) / h_{m}$.

The square root power-law dependence of $h_{m}$ on $L$ is in accord with expectations based on simple scaling theory. In the presence of more general intermolecular forces finite scaling suggests that for very wide stripes and away from co-existence the droplet height scales as $h_{m} \approx \delta \mu^{-\beta^{\mathrm{co}}} H\left(L / \xi_{\|}\right)$, where recall $\xi_{\|} \approx \delta \mu^{-v_{\|}^{\mathrm{co}}}$ is the complete wetting parallel correlation length. For fixed $L$ and $\delta \mu \rightarrow 0$ we require the scaling function vanishes as $H(x) \propto x^{\beta^{\text {co }} / \nu_{\|}^{\text {co }}}$ implying $h_{m} \propto L^{\frac{2}{p+2}}$ recovering Eq. (26) for the present case of van der Waals forces $(p=2)$.

For this model it is also possible to determine the equilibrium free-energy (per unit stripe length) of the droplet. Substituting the equilibrium profile back into Eq. (20) determines that

$$
F=\left(\gamma+W\left(h_{m}\right)\right) L+\sqrt{8 \gamma} \int_{h_{e}}^{h_{m}} d h \sqrt{W(h)-W\left(h_{m}\right)},
$$

where we have also included the extensive contribution $\gamma L$ coming from the interfacial tension. For the binding potential (22) the integral can be evaluated quite easily giving

$$
\begin{aligned}
F= & \left(\gamma+W\left(h_{m}\right)\right) L \\
& -\sqrt{8 \gamma A}\left[\sqrt{1-\frac{h_{e}^{2}}{h_{m}^{2}}}+\ln \left(\frac{h_{m}}{h_{e}}-\sqrt{\left.\frac{h_{m}^{2}}{h_{e}^{2}}-1\right)}\right) .\right.
\end{aligned}
$$

For large $L / h_{e} \gg 1$ this has the expansion

$$
F=\gamma L+\sqrt{2 A \gamma} \ln \frac{L}{h_{e}}+\cdots,
$$

where the higher-order terms are of order unity and also depend on $h_{e}, \gamma$, and $A$. The presence of the logarithmically diverging Casimir term means it is not possible to define a thermodynamic line tension associated with the three phase contact line for this system. Again this term is consistent with the anticipated finite-size scaling of the free-energy at complete wetting. Recall that for complete wetting at an infinite uniform planar wall, the wall-gas interfacial tension contains a singular contribution $\gamma_{\text {sing }} \approx \delta \mu^{2-\alpha_{s}^{c o}}$, where standard exponent relations determine that $2-\alpha_{s}^{c o}=1-\beta^{\mathrm{co}}[8]$. For a droplet of width $L$ we expect that this contribution scales as $\gamma_{\text {sing }} \approx \delta \mu^{2-\alpha_{s}^{c o}} B\left(L / \xi_{\|}\right)$with $B(x)$ a suitable scaling function. In the limit of $\delta \mu \rightarrow 0$ this implies that the freeenergy per unit length (along the $y$-axis) of the drop should contain a singular contribution $F_{\text {sing }} \propto L^{1-\left(2-\alpha_{s}^{c o}\right) / v_{\|}^{v_{0}^{\circ}}}$ which reduces to $F_{\text {sing }} \propto L^{(2-p) /(2+p)}$. This diverges as $L \rightarrow \infty$ for $p<2$ which is consistent with the marginal logarithmic divergence shown in Eq. (30) for the present case $p=2$. Similar logarithmic contributions occur in two dimensions for systems with short-ranged forces where they arise due to fluctuation effects. This similarity is not coincidental since in this dimension interfacial wandering leads to an effective entropic repulsion which also decays as an inverse square $[32,33]$.

\section{RESULTS}

In our DFT calculations we have considered the simplest case, setting $\varepsilon_{1}=0$, so that the outer material is modelled as a purely hard wall. This part of the substrate is therefore completely dry, with local contact angle $\theta_{1}=\pi$, at all (subcritical) temperatures. For the material of the stripe we set $\varepsilon_{2} \rho_{w}=1.2 \varepsilon \sigma^{-3}$ which, from earlier studies [34], we know leads to a first-order wetting transition occurring at $T_{w} / T_{c}=$ 0.83 were it of infinite extent. Here, $T_{c}$ is the bulk critical temperature which in our model occurs at $k_{B} T_{c} / \varepsilon=1.41$. Our present calculations are performed at $T / T_{c}=0.95$ far above the wetting transition ensuring that the local contact angle of the stripe phase $\theta_{2}=0$. This is also sufficiently below the critical temperature that the bulk correlation length is small, of order $\sigma$ [34]. Furthermore, we fix the chemical potential to its saturation value at this temperature, $\mu \equiv \mu_{\mathrm{sat}}=-3.945 \varepsilon$. For reference the coexisting bulk vapor and gas densities are $\rho_{v}=0.1308 \sigma^{-3}$ and $\rho_{l}=0.3872 \sigma^{-3}$, respectively. The Euler-Lagrange equation (19) was solved numerically on a two-dimensional rectangular grid with mesh spacing $0.1 \sigma$ (an order of magnitude smaller than the bulk correlation length) using standard Picard iteration. We considered a box of overall width $L_{x}=250 \sigma$ and height $L_{z}=30 \sigma$ and determined the equilibrium density profiles for different stripe widths $L=10 \sigma, 20 \sigma, \ldots, 200 \sigma$. As can be seen from the illustrative density profiles displayed in Fig. 2, a drop-like structure forms above the stripe for $L>20 \sigma$. For smaller widths only a microscopic film forms above the stripe and the mesoscopic interfacial Hamiltonian theory is not applicable. For larger widths however it is possible to extract the shape 

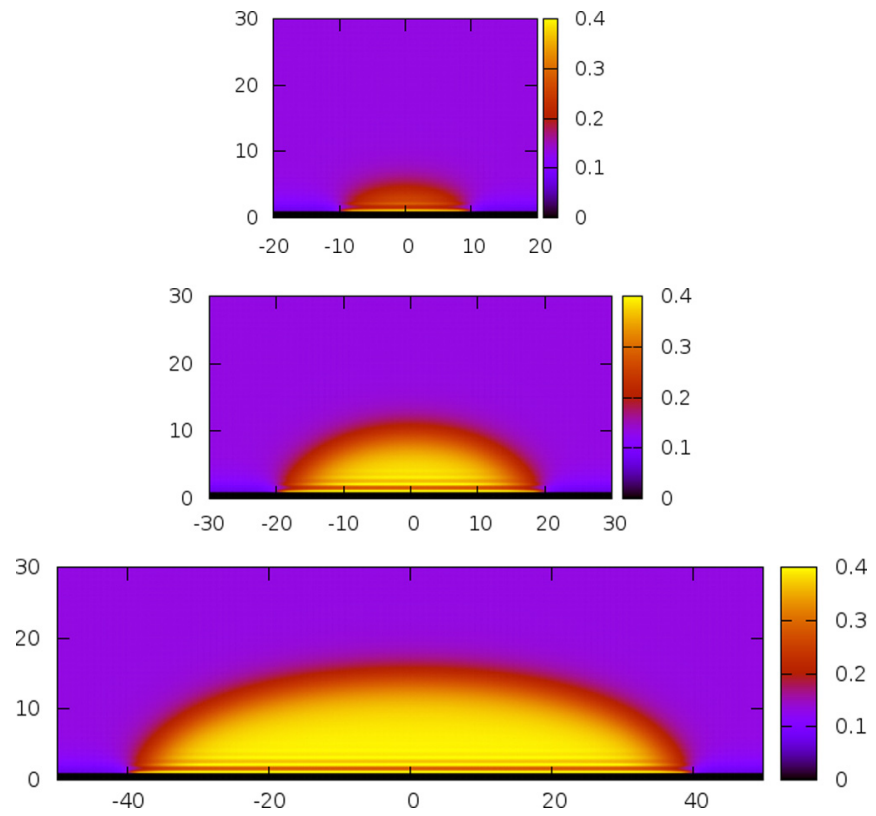

FIG. 2. Equilibrium density profiles as obtained from DFT at a temperature $T=0.95 T_{c}$ and bulk two-phase equilibrium for stripe widths (from top to bottom): $L=20 \sigma, L=40 \sigma$, and $L=80 \sigma$.

of an equilibrium drop $h(x)$ from the density profile $\rho(x, z)$ by tracing the contour of iso-density $\rho(x, h(x))=\left(\rho_{v}+\rho_{l}\right) / 2$. From this we can, in particular, extract the maximum (midpoint) height $\rho\left(0, h_{m}\right)=\left(\rho_{v}+\rho_{l}\right) / 2$. In Fig. 3 we show a log-log plot illustrating the dependence of $h_{m}$ on the stripe width $L$. For $L>40 \sigma$ there is evidently a linear dependence for which we find the slope $0.5079 \pm 0.01$ which is in excellent agreement with the prediction of Eq. (26) of the effective Hamiltonian theory. Interestingly, there is a sharp cross-over in the form of $h_{m}(L)$ at smaller values of $L$ which we discuss later.

Finally, in Fig. 4 we show our numerical results for the drop shape $h(x)$ in suitable dimensionless rescaled units $\tilde{x}=x / L$

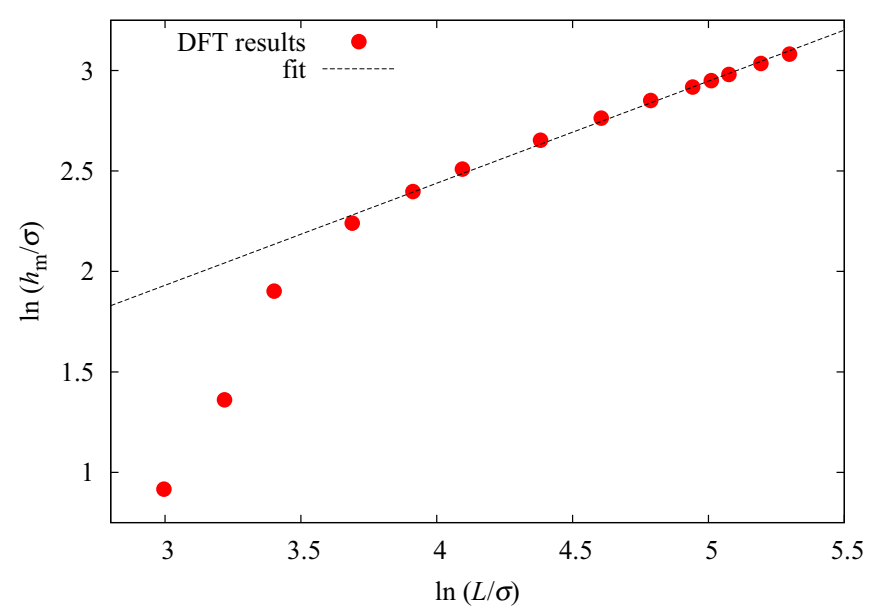

FIG. 3. Dependence of the drop height $h_{m}$ on the stripe width $L$. Symbols denote the DFT results with $h_{m}$ determined from the equilibrium density profile using the criterion $\left.\rho\left(0, h_{m}\right)=\left(\rho_{v}+\rho_{l}\right) / 2\right)$. The straight line fit to the data for $L \geqslant 40 \sigma$ has slope 0.5079 .

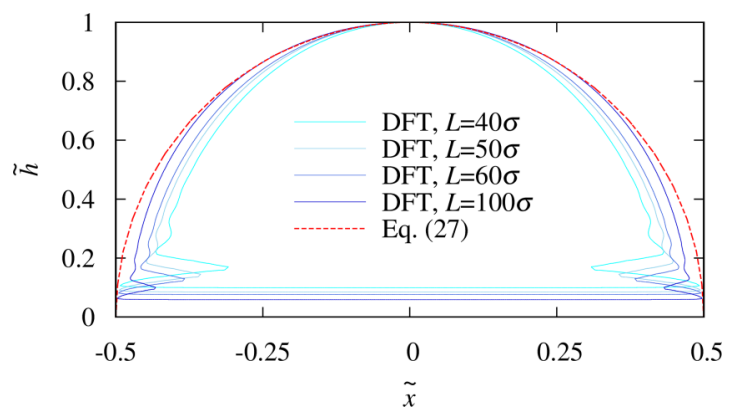

FIG. 4. DFT results for the droplet shape for different stripe widths $L$ in rescaled dimensionless units $\tilde{x}=x / L$ and $\tilde{h}(x)=$ $h(x) / h_{m}$. The dashed red line is the prediction of the effective Hamiltonian model.

and $\tilde{h}(x)=h(x) / h_{m}$ for different system sizes. While there is not perfect data collapse there is clear indication that even for relatively small system sizes the rescaled shape of the droplet is close to, and converging towards, the analytic result given by Eq. (27) (dashed red line).

\section{SUMMARY}

In this work we have used an accurate DFT based on a Rosenfeld-like fundamental measure theory model to study the formation and shape of liquid drops on a composite planar wall decorated by a single stripe of lateral width $L$ which is completely wet. For both the mid-point height and droplet shape we have found very good agreement with the predictions of effective Hamiltonian theory. Indeed we were surprised that the agreement is good even down to stripes which are only $50 \sigma$ across. This contrasts with the findings of DFT studies of capillary condensation where the agreement with the macroscopic Kelvin equation, for conditions where the walls which are completely wet, only occurs if the slit width is many hundreds of molecular diameters. This is indicative that mesoscopic effective Hamiltonians are, in general, reliable when the confining length scales are much larger than the underlying bulk correlation length (which is molecularly small in the present study). We were also intrigued to note that there appears to be a sharp cross-over in the dependence of $h_{m}$ on $L$ for $L<50 \sigma$. It may well be possible to explain this behavior by extending the simple interfacial model to allow for a position dependent binding potential $W(h ; x, L)$. Indeed within a sharp kink approximation, where we model the density profile as a sharp step function between the bulk liquid and gas, we can determine this from the external potential using $W(h ; x, L)=-\left(\rho_{l}-\rho_{v}\right) \int_{h}^{\infty} V(x, z) d z$. We can also improve on Eq. (20) by using the full expression for the interfacial area rather than a square gradient approximation. However, given that the resulting equations must be solved numerically they seem to offer little advantage over the full microscopic DFT calculation. Another point to note is that both approaches employed in this work were based on a meanfield approximation which neglects the effect of interfacial fluctuations. While these should not affect the scaling results for the size and shape of larger drops it is possible that they are important for smaller system sizes. Simulation studies of this would be very welcome. 
To finish we point out that new phenomena are likely to emerge when we consider walls decorated with multiple wetting stripes on a dry (or partially wet) surface. Consider for example the case of two parallel stripes of width $L$ separated by a distance $D$. When $D \gg L$ the stripes are essentially independent and isolated drops, similar to those described here, form above them. As the distance $D$ is decreased the two droplets must eventually coalesce to form a single drop containing a bubble of gas which spans the dry part of the substrate between the two stripes. This would be an example of a bridging transition akin to those studied for fluids between spheres and cylinders $[35,36]$. The distance $D_{B}$ at which this bridging transition occurs will be determined largely by the logarithmic Casimir interaction shown in Eq. (30) [37] and we anticipate $D_{B} \propto \ln L$. With three stripes bridging must also occur as we reduce the distance(s) between them but now we also have the possibility that the coalesced drop covers two or indeed three stripes. Similarly, it is natural to imagine that on a periodic array of stripes similar bridging transitions are induced by varying the inter-stripe gap. In this case such transitions must be accompanied by symmetry breaking since if we label the (wet) stripes $i=1,2,3,4, \ldots$ coalesced drops may span stripes 1 to 2,3 to 4 , etc., or (for exactly the same free-energy) 2 to 3,4 to 5 , etc. Indeed, it is possible that a sequence of symmetry breaking first-order transitions, producing larger coalesced droplets, is encountered as the distance between the stripes is reduced further. This may lead to very complex phase behavior even before the interplay with other surface phase transitions is allowed for. We hope that the present study is a first step to classifying these potentially very rich phase diagrams.

\section{ACKNOWLEDGMENTS}

This work was funded in part by the EPSRC UK Grant no. EP/L020564/1, "Multiscale Analysis of Complex Interfacial Phenomena". A.M. acknowledges the support from the Czech Science Foundation, Project No. 17-25100S.
[1] B. V. Derjaguin, Zh. Fiz. Khim. 137, 14 (1940).

[2] J. W. Cahn, J. Chem. Phys. 66, 3667 (1977).

[3] C. Ebner and W. F. Saam, Phys. Rev. Lett. 38, 1486 (1977).

[4] S. Dietrich, in Phase Transitions and Critical Phenomena, edited by C. Domb and J. L. Lebowitz (Academic Press, New York, 1988), Vol. 12.

[5] D. E. Sullivan and M. M. Telo da Gama, in Fluid Interfacial Phenomena, edited by C. A. Croxton (Wiley, New York, 1985).

[6] M. Schick, in Liquids and Interfaces, edited by J. Chorvolin, J. F. Joanny, and J. Zinn-Justin (Elsevier, New York, 1990).

[7] G. Forgacs, R. Lipowsky, and T. M. Nieuwenhuizen, in Phase Transitions and Critical Phenomena, edited by C. Domb and J. L. Lebowitz (Academic Press, London, 1991), Vol. 14.

[8] D. Bonn, J. Eggers, J. Indekeu, J. Meunier, and E. Rolley, Rev. Mod. Phys. 81, 739 (2009).

[9] R. Evans and P. Tarazona, Mol. Phys. 47, 1033 (1982).

[10] R. Lipowsky, Phys. Rev. Lett. 52, 1429 (1984).

[11] S. Y. Chou, P. R. Krauss, and P. J. Renstrom, Science 272, 85 (1996).

[12] M. Napiorkowski, W. Koch, and S. Dietrich, Phys. Rev. A 45, 5760 (1992).

[13] A. O. Parry, C. Rascón, and A. J. Wood, Phys. Rev. Lett. 85, 345 (2000).

[14] A. Malijevský and A. O. Parry, J. Phys.: Condens. Matter 25, 305005 (2013).

[15] M. Tasinkevych and S. Dietrich, Eur. Phys. J. E 23, 117 (2007).

[16] A. O. Parry, C. Rascón, N. B. Wilding, and R. Evans, Phys. Rev. Lett. 98, 226101 (2007).

[17] A. Malijevský and A. O. Parry, J. Phys.: Condens. Matter 26, 355003 (2014).
[18] C. Rascón and A. O. Parry, Phys. Rev. Lett. 94, 096103 (2005).

[19] C. Bauer, S. Dietrich, and A. O. Parry, Europhys. Lett. 47, 474 (1999).

[20] C. Bauer and S. Dietrich, Phys. Rev. E 61, 1664 (2000).

[21] C. Rascón, J. Phys.: Condens. Matter 12, A369 (2001).

[22] W. F. Saam, J. Low Temp. Phys. 157, 77 (2009).

[23] P. Yatsyshin, A. O. Parry, C. Rascón, and S. Kalliadasis, J. Phys.: Condens. Matter 29, 094001 (2017).

[24] A. O. Parry, A. Malijevský, and C. Rascón, Phys. Rev. Lett. 113, 146101 (2014).

[25] P. Lenz and R. Lipowsky, Phys. Rev. Lett. 80, 1920 (1998).

[26] H. Gau, S. Herminghaus, P. Lenz, and R. Lipowsky, Science 283, 486 (1999).

[27] A. O. Parry, E. D. Macdonald, and C. Rascón, J. Phys.: Condens. Matter 13, 383 (2001).

[28] Y. Rosenfeld, Phys. Rev. Lett. 63, 980 (1989).

[29] R. Evans, Adv. Phys. 28, 143 (1979).

[30] J. R. Henderson, in Fundamentals of Inhomogeneous Fluids, edited by D. Henderson (Dekker, New York, 1992).

[31] A. Malijevský, J. Phys.: Condens. Matter 25, 445006 (2013).

[32] D. B. Abraham, F. H. L. Essler, and A. Maciołek, Phys. Rev. Lett. 98, 170602 (2007).

[33] P. Jakubczyk, M. Napiorkówski, and A. O. Parry, Phys. Rev. E 74, 031608 (2006).

[34] A. Malijevský and A. O. Parry, Phys. Rev. Lett. 110, 166101 (2013).

[35] P. Hopkins, A. J. Archer, and R. Evans, J. Chem. Phys. 131, 124704 (2009).

[36] A. Malijevský and A. O. Parry, Phys. Rev. E 92, 022407 (2015).

[37] A. Malijevský and A. O. Parry (unpublished). 